\title{
Tuberous sclerosis complex: neonatal deaths in three of four children of consanguineous, non-expressing parents
}

Martino Ruggieri, Caterina Carbonara, Gaetano Magro, Nicola Migone, Sebastiano Grasso, Alessandra Tinè, Lorenzo Pavone, Manuel R Gomez

Division of Paediatric Neurology, Paediatric Clinic, University of Catania, I-95125 Catania, Italy

M Ruggieri A Tinè

L Pavone

CNR Immunogenetics and Experimental

Oncology, and

Department of

Genetics, Biology and

Medical Chemistry,

University of Turin,

I-10126 Turin, Italy

C Carbonara

N Migone

Institute of Anatomic Pathology, University of Catania, I-95100

Catania, Italy

G Magro

$S$ Grasso

Mayo Clinic, Mayo

Medical School,

Rochester, MN 55905, USA

M R Gomez

Department of Clinical

Genetics, Oxford

Radcliffe Hospital, Oxford OX3 7LJ, UK

M Ruggieri

Correspondence to: Dr Ruggieri, Oxford.

Received 29 April 1996 Revised version accepted for publication 25 October 1996

\begin{abstract}
We describe here four sibs, born to consanguineous, healthy, asymptomatic parents. Three of these infants had a rapidly fatal course in the neonatal period; death was attributed to congestive heart failure with radiographic evidence of cardiomegaly in all of them. Necropsy was done in only one of them and showed the typical findings of tuberous sclerosis complex (TSC) in the central nervous system (CNS), kidneys, heart, and liver. The fourth sib, currently 2 years old, also has typical signs of TSC, namely hypomelanotic skin macules and calcified subependymal nodules. Both parents and a living maternal grandmother had appropriate examination, which included skin inspection under Wood's lamp, dental examination, fundoscopy, echocardiography, abdominal and renal ultrasound, and head CT and MRI scans, and no signs of TSC were found in either parent or in the only living grandmother. By history alone there is no other relative with signs or symptoms suggestive of TSC. Linkage analysis and loss of heterozygosity (LOH) investigations on a variety of lesions obtained from postmortem and tissue or blood specimens from all available family members studied failed to identify a microdeletion in the chromosomal regions where TSC genes are located. It is very unusual that in a single TSC family there were three consecutive neonatal deaths, and very likely that all had cardiac rhabdomyomas. Moreover, to the best of our knowledge, there are no previous reports of TSC families with more than one affected sib, unusually severe manifestations of the disease, and completely normal, consanguineous parents.

(F Med Genet 1997;34:256-260)
\end{abstract}

Keywords: tuberous sclerosis; neonatal death; consanguinity.

TSC is an autosomal dominant condition characterised by the presence of hamartias and growth of hamartomas in one or more organs. ${ }^{1}$ Although CNS, skin, kidney, heart, retina, and lung are most commonly involved, nearly any organ in the body can be affected..$^{1-3}$ The disease prevalence is estimated at 1:6000 and two-thirds of cases are sporadic resulting from mutations. ${ }^{2-4}$

Linkage analysis of TSC families has shown there is genetic heterogeneity with two loci involved: TSC 1 is in chromosome region $9 \mathrm{q} 34^{5}$ and TSC2 is in 16p13.3. ${ }^{7}$ Recently, loss of heterozygosity has been reported in TSC hamartomas for TSC1 and TSC2 linked markers. $^{8}$

The number of families with defective TSC1 or TSC 2 genes is approximately the same ${ }^{9}$ and mutations of each of these two genes do not produce a distinct phenotype. ${ }^{10}$ There is a wide variation in expression within families ${ }^{124}$ and no instances of a "skipped" generation have been noted. ${ }^{11}$

We report on three sibs, born to consanguineous and healthy parents, all of whom had a rapidly fatal course and died in the early neonatal period from congestive heart failure. Necropsy showed in one of them the classical findings of TSC in the CNS, kidneys, liver, and spleen, and multiple rhabdomyomas of the heart; a fourth sib who is still alive had hypopigmented maculas and calcified subependymal nodules.

\section{Case reports}

PATIENT 1 (II.1, FIG 1)

A 3 day old male infant, the first born to first cousin parents, was admitted to the Paediatric Clinic of the University of Catania (PCUC), Italy, aged 3 days because of feeding difficulties, cyanotic attacks, and respiratory failure since birth. There was no history of familial disease. He was the term product of an uncomplicated pregnancy and was delivered by caesarean section because of fetal distress. Birth weight was $3500 \mathrm{~g}$, length $52 \mathrm{~cm}$, and head circumference $35 \mathrm{~cm}$.

Physical examination on admission showed severe and progressive respiratory failure with frequent apnoeic spells and generalised hypotonia. Pupillary and oculomotor reflexes were intact. Skin was normal. Chest radiograph showed cardiomegaly, which occupied most of the thoracic cavity. He died in heart failure at the age of 6 days.

Necropsy showed multiple cortical tubers and cardiac rhabdomyomas, histiocytosis of the spleen, and renal microcysts. The liver was slightly enlarged (154 g) and had a smooth capsular surface; the spleen was enlarged, firm in consistency, and weighed $25 \mathrm{~g}$.

Macroscopic examination of the brain disclosed typical firm, smooth tubers randomly 

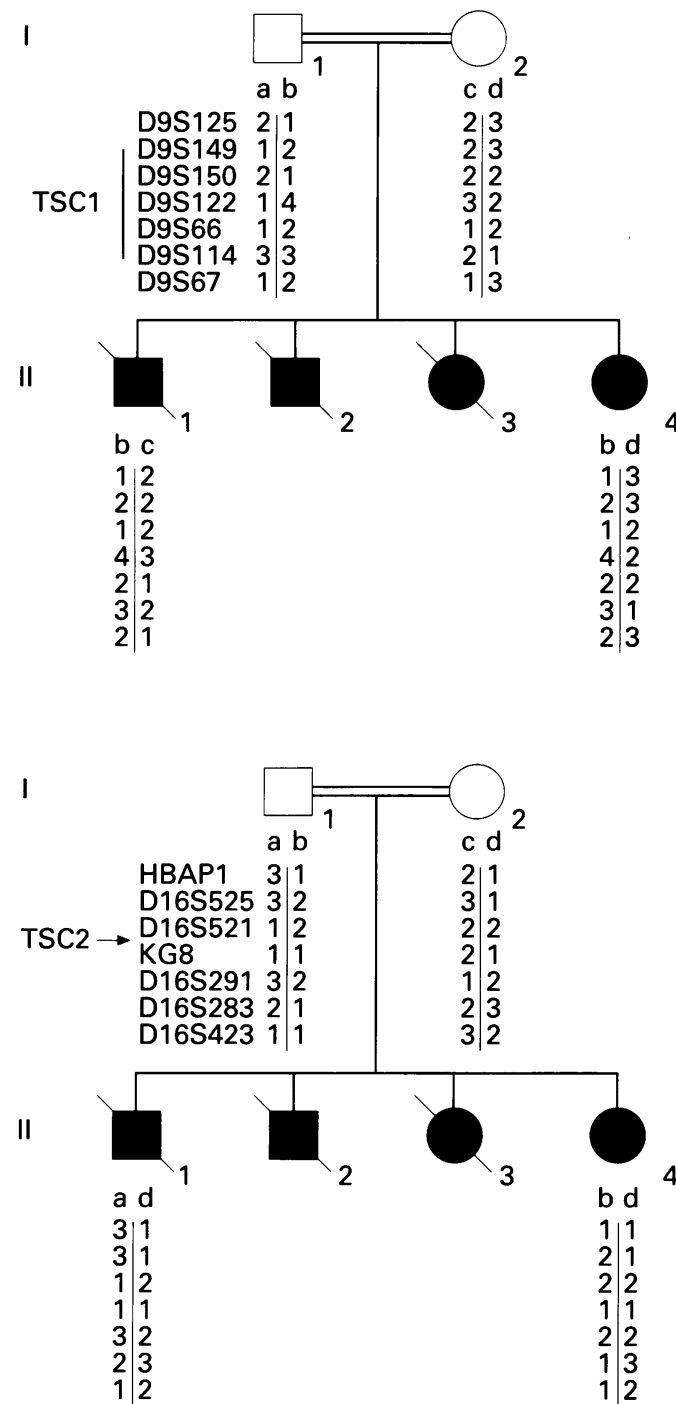

Figure 1 Pedigree of family. Segregation analysis of TSC1 (upper panel) and TSC2 (lower panel) linked polymorphic markers. The solid bar on the left of the polymorphic markers shows the TSC1 critical region (between D9S149 and D9S114). Parental haplotypes are indicated by lower case letters $(a-d)$.

distributed over both cerebral hemispheres, with frontal lobe predominance. The heart displayed several white to yellow-tan intramural tumours, a few millimeters to several centimeters in diameter, irregularly shaped but well demarcated from the surrounding myocardium, protruding over the epicardium and the endocardium to occupy the cardiac chambers (fig 2). Sectioning of the liver showed a normal, brownish-tan lobular architecture; sectioning of the spleen showed an irregular surface with numerous nodules measuring $8 \mathrm{~mm}$ in diameter, raised above the surrounding parenchyma. Renal examination showed multiple bilateral microcysts throughout the parenchyma, involving both cortex and medulla.

Microscopic examination of the brain showed conspicuous histological disorganisation with disruption of the normal cortical pattern, paucity of neurones, increased astrocytic nuclei, and the presence of large dysplastic neurones. Light microscopy of the myocardium showed circumscribed but not capsulated clusters of glycogen filled myocytes (fig 3 ). The liver was disorganised in some places by the presence of groups of a few to as many as 50 rounded hepatocytes, $80 \mathrm{~mm}$ in diameter, containing one or two vesicular nuclei with a prominent nucleolus and eosinophilic cytoplasm. Irregularly shaped hepatocytes were extremely vacuolated (fig 4). Around these groups of atypical hepatocytes, the lobular architecture was preserved. Twelve of these lesions were found in 15 paraffin blocks of the liver that measured $10 \times 15 \times 4 \mathrm{~mm}$. The spleen architecture was formed by large clusters of brownish connective tissue supporting thin walled blood vessels along with scattered red blood cells, lymphocytes, plasma cells, and numerous, large, haemosiderin laden PAS positive histiocytes, some of which contained two to eight vesicular nuclei with a prominent nucleolus. Most of the foci were poorly delimited from the adjoining splenic parenchyma; the uninvolved red pulp was congested. The renal microcysts had a characteristic lining of hyperplastic epithelium formed by large cells with acidophilic cytoplasm (fig 5).

PATIENT 2 (II.2)

A male infant, brother of II.1, product of an uncomplicated term pregnancy, was also delivered by caesarean section because of fetal distress. Birth weight was $3300 \mathrm{~g}$, length 53 $\mathrm{cm}$, and head circumference $35 \mathrm{~cm}$. His cry was feeble and suck almost absent.

On day 1 he had periodic breathing, severe hypotonia, and a feeble cry. The skin was normal. In the following hours he went into respiratory failure and chest radiographs showed cardiomegaly. $\mathrm{He}$ died in congestive heart failure that day. Necropsy was not performed.

PATIENT 3 (II.3)

A 4 day old female infant, sister of II. 1 and II. 2 and the product of an uncomplicated term pregnancy, was also delivered by caesarean section because of fetal distress. Birth weight was $3400 \mathrm{~g}$, length $52 \mathrm{~cm}$, and head circumference $34.5 \mathrm{~cm}$. Cry and suck were normal at birth. Apgar scores were 7 and 9 at one and five minutes.

On admission to a Perinatal Care Unit elsewhere in Italy, she was found to be in congestive heart failure. A chest radiograph showed, as in her sibs, marked cardiomegaly. She also died in cardiac failure but four days after birth. No necropsy was performed.

PATIENT 4 (II.4)

A 24 month old female, sister of patients 1,2 , and 3, was born at term and delivered by caesarean section. Pregnancy was complicated by transient hyperglycaemia and treated with insulin. Birth weight was $3080 \mathrm{~g}$, length $50 \mathrm{~cm}$, and head circumference $35 \mathrm{~cm}$. At 3 days of age she presented with apnoeic spells and was admitted to an Intensive Care Unit elsewhere in Italy. During such crises only a slight increase in the plasma ammonium (223 $\mathrm{mg} / 100 \mathrm{ml}$, normal values $<200$ ) was detected, but within 24 hours was within the normal range. Extensive metabolic screening was normal during the apnoeic spells. EEG, echocardiogram, abdominal and pelvic ultrasonogra- 
A
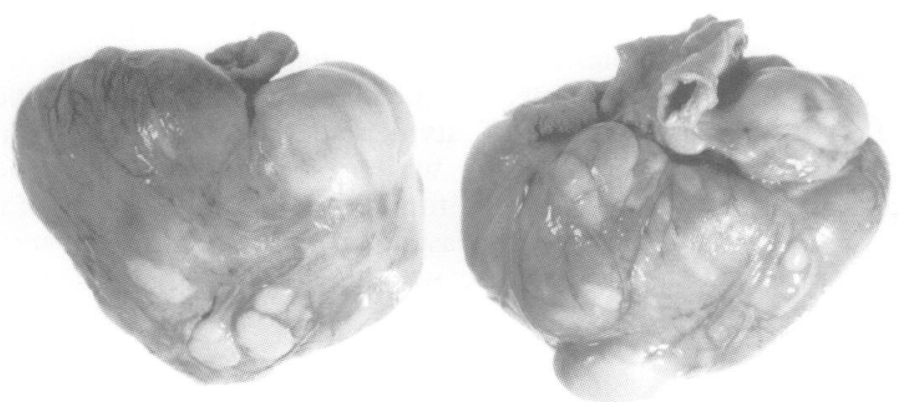

$\begin{array}{lllllllllllllllll}2 & 3 \text { fF } & 4 & 5 & 6 & 7 & 8 & 9 & 10 & 11 & 12 & 13 & 14 & 15 & 16 & 17\end{array}$

B
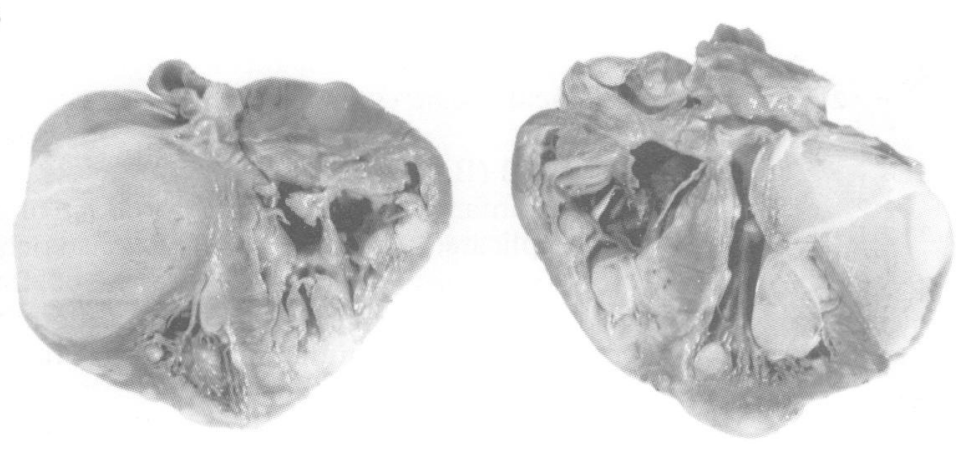

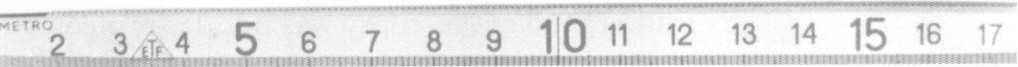

Figure 2 Patient 1 (II.1). (Top) Lateral view of the heart; the tumours form white-tan lesions that vary from several millimeters to several centimeters. They are irregularly shaped and appear well demarcated from the surrounding myocardium. (Bottom) Open atrial and ventricular cavities showing large intracavity tumours arising from the anterior and lateral walls and protruding into the cavities. Note that the largest masses produce left and right ventricular outflow tract obstruction.

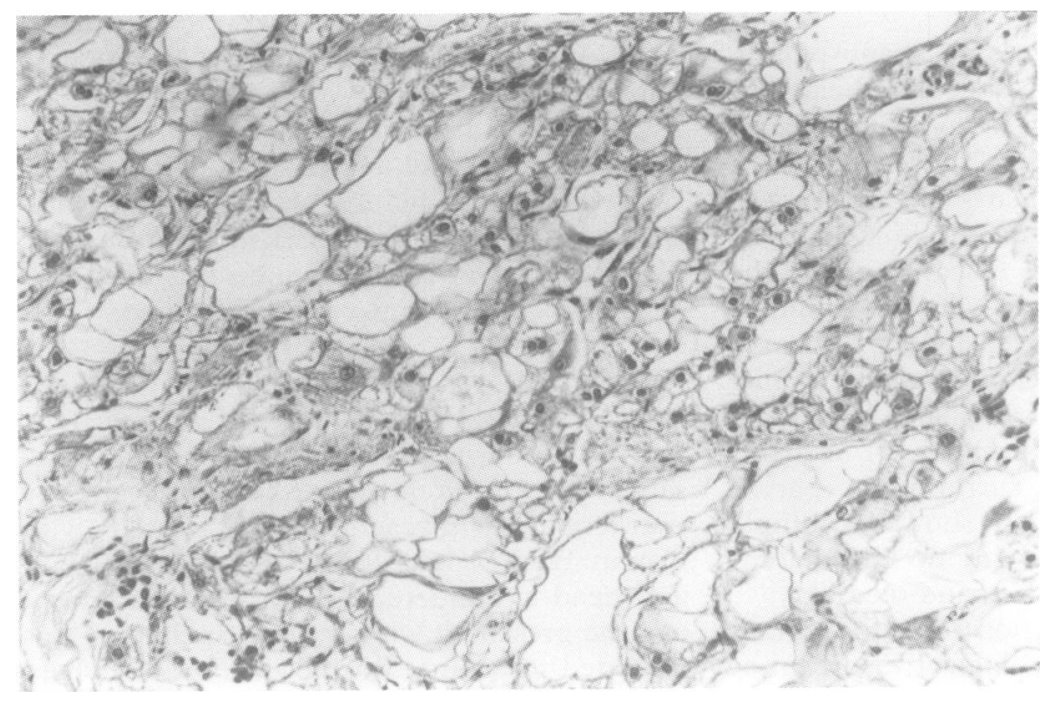

Figure 3 Patient (II.1). Microscopic section of one of the tumours shown in fig 2 show the large clear rhabdomyoma cells with the spongy vacuolated appearance.

phy, and cerebral ultrasonography were all normal. At birth examination of the skin with Wood's lamp showed white spots but the same examination at 24 months disclosed four hypo- pigmented macules. Fundoscopy after mydriasis showed no hamartomas. Routine blood and urine investigations were within the normal range. There was slight generalised hypotonia but no other findings on physical examination. EEG and cardiac, abdominal, and pelvic ultrasound investigations were all normal. Head CT scan showed several calcified lesions in the wall of the left lateral ventricle and in the right foramen of Monro.

Examination of both parents and the maternal grandmother including inspection of the skin under Wood's light, oral cavity, and eyegrounds were negative. EEG, ECG, echocardiography, abdominal and pelvic ultrasonography, and cerebral contrast enhanced CT and MRI scans were normal. There was no family history suggestive of TSC.

\section{Molecular analysis}

DNA from the parents and their daugther (II.4) was extracted from peripheral blood leucocytes. DNA from the first son (II.1) was obtained from histological sections of a cortical tuber and, as control, of the surrounding normal tissue embedded in paraffin. Linkage analysis at the two TSC loci was attempted by means of a total of 14 microsatellite markers located at 9q34 and 16p13.3 (fig 1). The two sibs showed different haplotype combinations at both loci. The linkage data were compatible with either a TSC1 or TSC2 gene defect. Furthermore, the consanguineous parents did not share any 9 q34 or 16 p13.3 haplotype identical by descent. A cortical tuber sample obtained at necropsy of II. 1 was investigated for $\mathrm{LOH}$ at 12 informative markers spanning the TSC1 and TSC2 regions (D9S149, D9S150, D9S122, D9S66, D9S114, D9S67 and HBAP1, D16S525, D16S521，D16S291，D16S283, D16S423, respectively). The germline heterozygosity was mantained in all $9 \mathrm{q} 34$ and 16 p13.3 loci.

\section{Discussion}

Three sibs died in the neonatal period in acute cardiac failure; postmortem examination of II. 1 showed multiple cerebral tubers, intracavity cardiac rhabdomyomas, renal microcysts, and histiocytosis of the liver and spleen, leaving no doubt of the diagnosis of tuberous of sclerosis. ${ }^{1-3}$ Two other sibs (II.2 and II.3) died $N$ in heart failure in the first four days of life and N had two direct relatives with TSC (II.1 and II.4). Patient II.1 died from heart failure resulting from obstructive intracavity cardiac tumours, specially the large rhabdomyoma in the right lower chamber (fig 2). The two sibs (II.2 and II.3) who did not have necropsy also presented with cardiac failure and extreme cardiomegaly and probably had intracavity rhabdomyomas. It is very unusual that in a single family there were three consecutive neonatal deaths and very likely that all had cardiac rhabdomyomas.

The mortality rates among patients with cardiac rhabdomyomas and TSC compared to those with cardiac masses without TSC are different. In the large majority ( $>80 \%$ ) of TSC patients diagnosed at birth with cardiac 


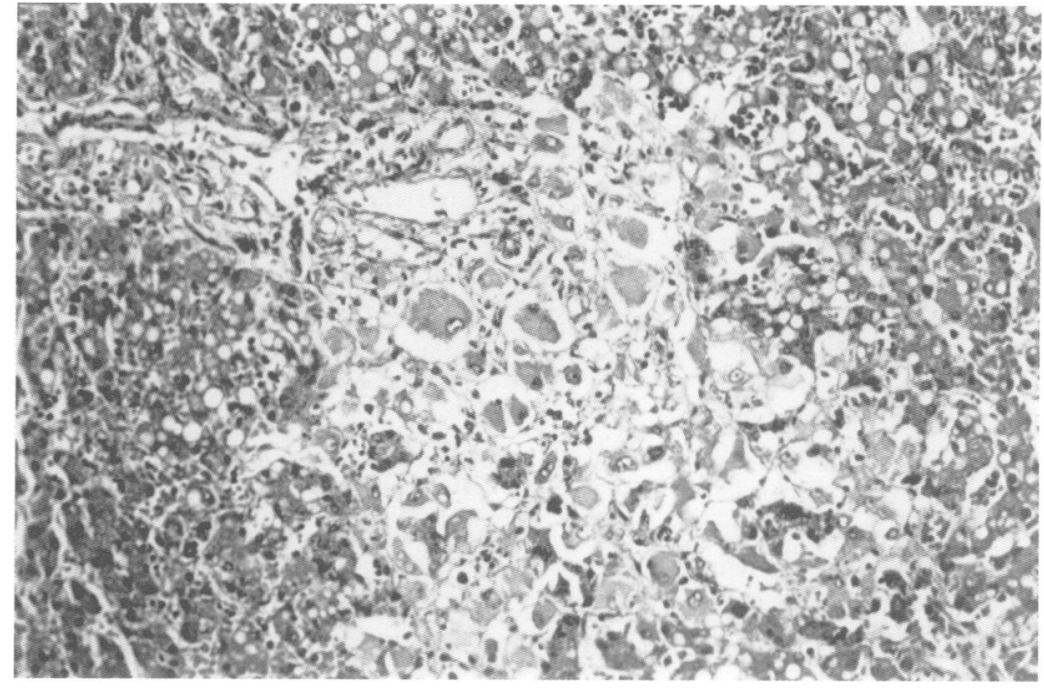

Figure 4 Patient (II.1). Group of large, distorted, vacuolated hepatocytes which resemble the "spider cells" seen in the cardiac rhabdomyoma (haematoxylin-eosin).

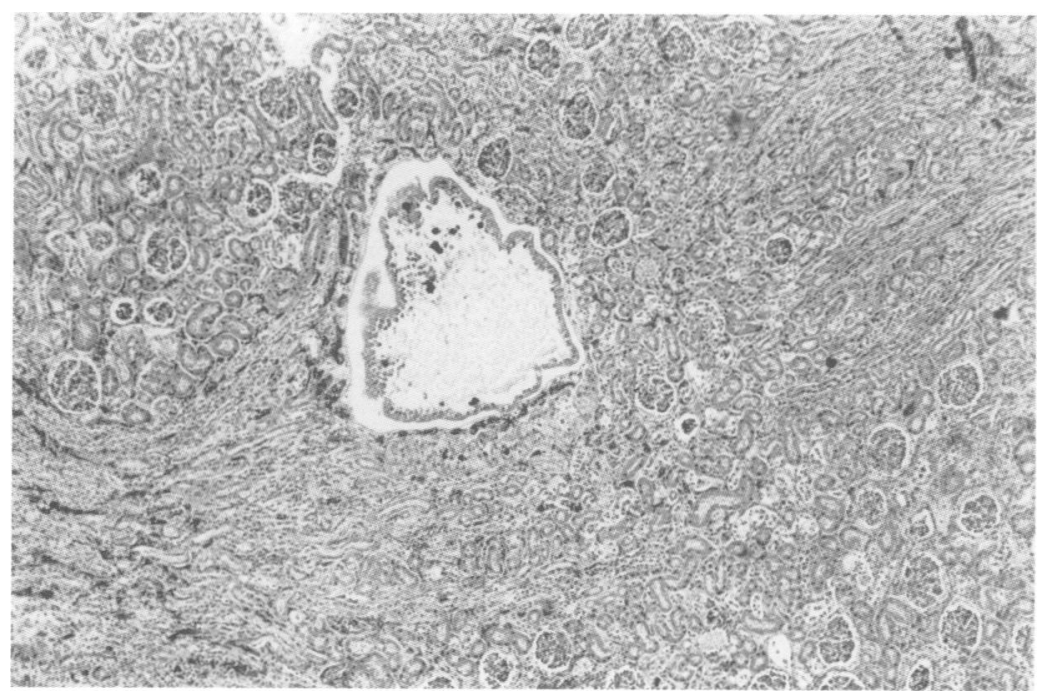

Figure 5 Patient 1 (II.1). A cyst in the renal parenchyma with evidence of concentrically oriented spindle cells surrounding multiple small tubular lumina lined with hyperplastic epithelium (haematoxylin-eosin).

rhabdomyomas, there are no symptoms and the tumours regress during infancy, ${ }^{12-15}$ whereas in the absence of other TSC signs the rhabdomyomas often present as large intracavity tumours and have a worse prognosis. ${ }^{15-17} \mathrm{~A}$ bias in the manner of ascertainment of cardiac signs (primary in non-TSC, often secondary in TSC patients) may account for this difference. A second possibility is that the non-TSC associated rhabdomyomas may have a different pathogenetic mechanism. The three neonatal deaths in the TSC family described had first cousin parents. It is thus possible that a recessive mutation might have contributed to the recurrence of severe cardiac involvement in the present family, or even to the occurrence of other genetic diseases (for example, metabolic) commonly associated with cardiac involvement. ${ }^{18} 19$ So far, familial rhabdomyomas segregating independently from TSC have not been reported. Moreover, without pathological evidence of cardiac rhabdomyomas in II. 2 and II.3, we have to consider other possible causes of congestive heart failure in the term neonate, namely, asphyxial cardiomyopathy, arteriovenous malformations, left sided obstructive lesions, large mixing cardiac defects, or even viral myocarditis. ${ }^{20}$ It is well known that family clusters of congenital heart disease are not uncommon. ${ }^{19}$ It is indeed possible that the three infants who died inherited a mutation of the TSC1 or TSC2 gene from each parent while the living child inherited only one mutated TSC gene from one of the parents. It could be, as in the Eker rat, that the homozygous state is lethal ${ }^{21}$ and the heterozygous state is associated with renal cell carcinoma. However, it is hard to conceive that both parents are carriers of a TSC mutation and do not express any signs or that both have germline and not somatic mutations. The parents did not share a common haplotype either at the TSC1 locus or at TSC2.

The family represents an example of a high degree of penetrance of the TSC gene in three sibs, with intrafamilial variability only in the fourth sib. The multiple neonatal deaths possibly resulting from intracavity cardiac rhabdomyomas contrast with the non-expression of presumably heterozygous parents and the reduced expression in the only living sib who may also be heterozygous.

Linkage analysis of 9q34 and 16p13.3 and $\mathrm{LOH}$ investigation on a variety of pathological tissues were attempted to identify which TSC locus was involved. A definitive locus assignment could not be reached by either method. All tissue samples available from II.1 (rhabdomyomas, cortical tubers, and kidney hamartomas) were obtained postmortem, which may account for finding clearly reproducible microsatellite patterns only in a few samples. Overall, the molecular data were compatible with either a TSC1 or TSC2 mutation transmitted by either of the parents.

Although there is no clear evidence for the existence of a third locus for TSC, ${ }^{8-10}$ further linkage analysis is under way in this family in search for a different locus for TSC.

Possible explanations for this unusual family history include: (1) a recessive form of tuberous sclerosis; (2) non-penetrance or incomplete penetrance of the disease in one of the parents; (3) gonadal mosaicism in one of the parents; (4) a new mutation in each child. The latter is extremely unlikely. Although there is no compelling evidence that TSC can be inherited as anything but an autosomal dominant disorder, the suggestion that the few instances of affected sibs with apparently normal parents, and our cases with consanguineous non-expressing parents, may result from an autosomal recessive gene defect with a similar clinical phenotype is theoretically possible, and could account for the marked intrafamilial heterogeneity. ${ }^{1}$ By contrast, there are no previous published reports, as far as we know, of TSC families with more than one affected sib, unusually severe manifestations of the disease, and completely normal consanguineous parents. However, more plausible explanations exist. It has been long recognised that expression is highly variable and minor manifestations may be missed in mildly affected subjects. ${ }^{1-3}$ There have been few reports of 
obligate gene carriers for TSC with two affected children, who on dermatological and eye examination, ultrasound investigations, and CT scan had no evidence of the disease, ${ }^{22}$ and examples where a single lesion was the only manifestation of TSC. ${ }^{23}$ In the present family a thorough examination of both parents, including imaging of the brain and kidneys did not show any signs of TSC. The best explanation for the absence of signs in both parents of two or more affected children seems to be gonadal mosaicism without mosaicism of somatic cells. To our knowledge gonadal mosaicism has not yet been proven in TSC; however, somatic mosaicism was recently described ${ }^{24}$ in a nuclear family where the father of a 2 year old boy with proven TSC had subclinical signs of TSC and an apparently low proportion of cells with the TSC2 mutation.

A further point deserves comment, namely the anomalous liver and splenic lesions in patient II.1. Liver and spleen involvement has been regarded for many years as an unusual finding in TSC. ${ }^{125}$ However, several patients with TSC and involvement of the liver, spleen, or both, have been reported. ${ }^{125}$ The few lesions studied from postmortem material were most often asymptomatic angiomyolipomas and rarely neurilemmoblastomas, lipomesenchymal tumours, hamartomas, lipomas, and marked fibrosis of the portal spaces in the liver, ${ }^{125}$ and angiomas or hamartomas in the spleen. ${ }^{1}$ Some hepatic lesions had a microscopic appearance similar to polycystic disease of the liver, others contained fusiform cells resembling smooth muscle cells or foci of proliferating blood vessels of capillary size; the splenic hamartomas were mostly characterised by large haemosiderin laden histiocytes. ${ }^{1}$ The foci of large, bizarre hepatocytes seen in patient II.1, previously reported by one of us (SG), ${ }^{26}$ were reminiscent of the lesions of the brain, heart, and spleen. As in rhabdomyomas, a few of these hepatocytes looked like "spider cells". ${ }^{26}$ To the best of our knowledge, there are no previous descriptions of hepatic abnormality identical to this. ${ }^{125}$

The authors wish to thank Dr Bernd Scheithauer (Rochester USA) for his valuable support in reviewing the original brain lides of patient II.1, and Dr Susan M Huson (Oxford, UK) fo her valuable suggestions and helpful discussion. Financial support from Telethon Italy, Grant No E-143, and from Associazione Emma ed Ernesto Rulfo per la Genetica Medica is gratefully aknowledged. We wish to thank the Italian Lay Group "Associazione Italiana NeuroEctodermosi (AINE) - Sicilia Orientale" for their valuable support.
1 Gomez MR. Tuberous sclerosis. 2nd ed. New York: Raven Press, 1988.

2 Webb DW, Osborne JP. Tuberous sclerosis. Arch Dis Child 1995;70:471-4.

3 Gomez MR. Phenotypes of the tuberous sclerosis complex with a revision of diagnostic criteria. Tuberous sclerosis and allied disorder. Ann N Y Acad Sci 1991;615:1-7.

4 Ahlsen G, Gillberg C, Linbdolm R, Gillberg G. Tuberous sclerosis in western Sweden. A population study of cases $\mathbb{D}$ with early childhood onset. Arch Neurol 1994;51:67-72.

5 Fryer AE, Chalmers A, Connor JM, et al. Evidence that the $\mathbb{8}$ gene for tuberous sclerosis is on chromosome 9. Lancet 1987;1:659-61.

6 Kandt RS, Haines JL, Smith M, et al. Linkage of an important gene locus for tuberous sclerosis to chromosome 16 marker for polycystic kidney disease. Nat Genet 1992;2:3741.

7 European Chromosome 16 Tuberous Sclerosis Consortium. Identification and characterization of the tuberous $\bar{\omega}$ sclerosis gene on chromosome 16. Cell 1993;75:1305-9.

8 Carbonara C, Longa L, Grosso E, et al. Preferential loss of heterozygosity at TSC2 over TSC1 chromosomal region in tuberous sclerosis hamartomata. Genes Chrom Cancer 1996;15:18-25.

9 Povey S, Burley MW, Attwood J, et al. Two loci for tuberous sclerosis: one on $9 \mathrm{q} 34$ and one on 16p13. Ann Hum Genet 1994;58:107-27.

10 Wilson PJ, Ramesh V, Kristiansen A, et al. Novel mutations detected in the TSC2 gene from both sporadic and familial TSC patients. Hum Mol Genet 1996,5:249-56.

11 Webb DW, Osborne JP. Non-penetrance in tuberous sclerosis. F Med Genet 1991;28:417-19.

12 Shepherd CW, Gomez MR, Lie JT, Crowson CS. Causes of death in patients with tuberous sclerosis. Mayo Clin Proc 1991;66:792-6.

13 Webb DW, Osborne JP. Cardiac rhabdomyomas and their association with tuberous sclerosis. Arch Dis Child 1993;68: 367-70

14 Amiram N, Tajik AJ, Freeman WK, et al. Tuberous sclerosi and cardiac rhabdomyoma. Am $\mathcal{F}$ Cardiol 1995;76:419-21.

15 Harding CO, Pagon RA. Incidence of tuberous sclerosis in patients with cardiac rhabdomyomas. $A m \mathcal{F}$ Med Genet 1990;37:443-6.

16 Fenoglio JJ, McAllister HA, Ferrans VJ. Cardiac rhabdomyoma: a clinicopathological and electron microscopic study. Am F Cardiol 1976;38:241-51.

17 Smythe JF, Dyck JD, Smallhorn JF, Freedom RM. Natura history of cardiac rhabdomyoma in infancy and childhood. Am $\mathcal{F}$ Cardiol 1990;72:211-15.

18 Bulbul ZR, Rosenthal D, Brueckner M. Genetic aspects of heart disease in the newborn. Semin Perinatol 1993;17:6175.

19 Nora JJ, Berg K, Nora AH. Cardiovascular diseases. Genetics, epidemiology and prevention. Oxford: Oxford University Press, 1991.

20 Bernstein D. Congestive heart failure. In: Behrman RE Kliegman RM, Arvin AM, eds. Nelson textbook of pediatrics. 15th ed. Philadelphia: W B Saunders, 1996:1359-64.

21 Kobayashi T, Hirayama Y, Kobayashi E, Kubo Y, Hino O. A germline insertion in the tuberous sclerosis (Tsc2) gene gives rise to the Eker rat model of dominantly inherited cancer. Nat Genet 1995;9:70-4.

22 Bundey S. Phacomatoses and tumours. In: Bundey S, ed Genetics and neurology. 2nd ed. Edinburgh: Churchill Livingstone, 1992:37-66.

23 Zeller J, Friedman D, Clerici T, Revuz J. The significance of periungual fibroma: report on seven cases. Arch Dermatol 1995;131:1465-6.

24 Verhoef S, Vrtel R, van Essen T, et al. Somatic mosaicism and clinical variation in tuberous sclerosis complex. Lancet 1995;345:202.

25 Jozwiack S, Pedich M, Rajszys P, Michalowicz R. Incidence of hepatic hamartomas in tuberous sclerosis. Arch Dis Child 1992;67:1363-5.

26 Grasso S, Manusia M, Sciacca F. Unusual liver lesion in tuberous sclerosis. Arch Pathol Lab Med 1982;106:49. 